\title{
Effect of SMYD3 on the microRNA expression profile of MCF-7 breast cancer cells
}

\author{
DONGJU CHEN ${ }^{1,2^{*}}$, LEI LIU ${ }^{1,2^{*}}$, XUEGANG LUO $^{1,2^{*}}$, AI MU $^{1,2}$, LIHUA YAN $^{1,2}$, XIAOYING CHEN $^{1,2}$, \\ LEI WANG ${ }^{1,2}$, NAN WANG ${ }^{1,2}$, HONGPENG HE ${ }^{1,2}$, HAO ZHOU ${ }^{1,2}$ and TONGCUN ZHANG ${ }^{1,2}$ \\ ${ }^{1}$ Key Laboratory of Industrial Fermentation Microbiology of the Ministry of Education, Tianjin University of Science \\ and Technology; ${ }^{2}$ Tianjin Key Laboratory of Industrial Microbiology, College of Biotechnology, \\ Tianjin University of Science and Technology, Tianjin 300457, P.R. China
}

Received September 15, 2015; Accepted March 28, 2017

DOI: $10.3892 / 01.2017 .6320$

\begin{abstract}
SET and MYND domain containing 3 (SMYD3) is a histone methyltransferase (HMT) and transcription factor, which serves important roles in carcinogenesis. Numerous downstream target genes of SMYD3 have been identified in previous studies. However, the downstream microRNA (miRNA) s regulated by SMYD3 are yet to be elucidated. In the present study, the results of miRNA microarray demonstrated that 30 miRNA expression profiles were upregulated, whilst 24 miRNAs were downregulated by $>2.0$-fold in the SMYD3-overexpressed MCF-7 breast cancer cells. The HMT activity was demonstrated to be essential for SMYD3-mediated transactivation of miR-200c-3p and the overexpression of miR-200c-3p inhibited the transactivation effects of SMYD3 on myocardin-related transcription factor-A-dependent migration-associated genes. To our best knowledge, the current study is the first to report on the transcriptional regulation of SMYD3 on miRNAs, and miR-200c may be a downstream negative regulator of the SMYD3-mediated pathway in the migration of breast cancer cells. These results may provide a novel theoretical basis to understand the mechanisms underlying the initiation, progression, diagnosis, prevention and therapy of breast cancer.
\end{abstract}

\section{Introduction}

SET and MYND domain containing 3 (SMYD3) is a novel histone methyltransferase gene identified in hepatoma and

Correspondence to: Professor Xuegang Luo, Key Laboratory of Industrial Fermentation Microbiology of the Ministry of Education, Tianjin University of Science and Technology, 29 13th Street, Tianjin 300457, P.R. China

E-mail: luoxuegang@hotmail.com

*Contributed equally

Key words: SET and MYND domain containing 3, microRNA, breast cancer, miR-200c, miR-149 colon carcinoma cells by Hamamoto et al (1). SMYD3 is located on human chromosome 1 and encodes two protein isoforms that are composed of 428 and 369 amino acids. Previous studies have demonstrated that SMYD3 is frequently overexpressed in numerous types of cancer cells, including hepatic, colon, gastric and cervical carcinoma, and breast cancer (2-4), whilst the expression levels were lower in the corresponding normal tissue. A number of previous studies have demonstrated that SMYD3 has vital roles in the process of tumor development via its functions as a histone methylation enzyme and a transcription factor $(5,6)$. SMYD3 modifies chromatin structure by catalyzing the methylation of histone H3 at lysine 4 (H3K4), H4K20 and H4K5 $(5,6)$. Also, SMYD3 regulates the transcription of target genes via associating with RNA polymerase II or HELZ RNA helicase and binding at the motif CCCTCC or GGAGGG in the promoter (1).

MicroRNAs (miRNAs) are small, non-coding, endogenous RNA molecules of 18-22 nucleotides that were first identified in Caenorhabditis elegans. miRNAs suppress gene expression by binding the targeted mRNA transcripts, which causes translational repression or mRNA degradation. Previous studies demonstrated that miRNAs serve important roles in tumorigenesis through the regulation of genes involved in cancer development and maintenance $(7,8)$.

A number of studies have identified that histone methylation and miRNAs are essential in the initiation and progression of cancer (7-9). However, the association between SMYD3 and miRNAs is yet to be elucidated. To investigate this further, the current study analyzed the global regulatory effects of SMYD3 on miRNAs in breast cancer cells using miRNA microarrays and reverse transcription-quantitative polymerase chain reaction (RT-qPCR).

\section{Materials and methods}

Cell lines and plasmids. The MCF-7 human breast cancer cell line was obtained from the American Type Culture Collection (Manassas, VA, USA). The plasmid pcDNA5-TO/TAP-DEST-SMYD3 was a gift from Professor Philip Tucker from the Institute for Cellular and Molecular Biology, University of Texas (Austin, TX, USA). The CON049 (GeneChem, Co., Ltd., Shanghai, China) plasmid was used as 
a negative control for short hairpin (sh)SMYD3 (GeneChem, Co., Ltd.). The SMYD3- $\Delta$ NHSC plasmid was constructed by deleting the 205-208th amino acids (Asn-His-Ser-Cys) of pcDNA5-TO/TAP-DEST-SMYD3. The miR-200c-3p mimic and its negative control were obtained from Qiagen, Inc., (Valencia, CA, USA).

Cell culture and transfection. The MCF-7 cells were cultured in Dulbecco's modified Eagle's medium (DMEM/F-12; Gibco; Thermo Fisher Scientific, Inc., Waltham, MA, USA) supplemented with $10 \%$ fetal bovine serum (Tianjin Kangyuan Biotechnology Co., Ltd., Beijing, China) at $37^{\circ} \mathrm{C}$ in an atmosphere containing $5 \% \mathrm{CO}_{2}$. For plasmid transfection experiments, MCF-7 cells were cultured in DMEM/F-12 medium without added hormones at $60 \%$ confluence for $12 \mathrm{~h}$, and then transfected using TurboFect ${ }^{\mathrm{TM}}$ in vitro transfection reagent (Roche Diagnostics, Indianapolis, IN, USA) according to the manufacturer's protocol. Following incubation for $6 \mathrm{~h}$, the medium was removed and replaced with normal culture medium (DMEM/F-12 medium without hormones) for $24 \mathrm{~h}$. Then, the RT-qPCR was performed as described below. For miR-200c-3p mimic transfection experiments, Lipofectamine ${ }^{\circledR} 2000$ reagents (Invitrogen; Thermo Fisher Scientific, Inc.) were used following the manufacturer's protocol.

RNA extraction and complementary DNA (cDNA) synthesis. Briefly, total cellular RNA was extracted from cultured cells using TRIzol reagent according to the manufacturer's protocol (Invitrogen; Thermo Fisher Scientific, Inc.) and $2 \mu \mathrm{g}$ total RNA was reverse-transcribed using M-MLV reverse transcriptase (Promega Corporation, Madison, WI, USA) according to the manufacturer's protocols. miRNA was isolated using the miRcute miRNA isolation kit (\#DP501; Tiangen Biotech, Co., Ltd., Beijing, China), followed by cDNA synthesis using SuperScript First-Strand Synthesis system (Invitrogen; Thermo Fisher Scientific, Inc.), but with the specific stem loop primer primers. The miRNA RT-PCR primer sequences were as follows: JH6-miR200c-3p, 5'-CTCAACTGGTGTCGTGGAG TCGGCAATTCAGTTGAGTCCATC-3'; JH7-miR200c-3p, 5'-CTCAACTGGTGTCGTGGAGTCGGCAATTCAGTTGA GTCCATCA-3'; JH7-miR 149-3p, 5'-CTCAACTGGTGTCGTG GAGTCGGCAATTCAGTTGAGGGGAGTG-3'. The total RNA and dNTPs were incubated at $65^{\circ} \mathrm{C}$ for $5 \mathrm{~min}$, cooled on ice briefly. Subsequently, the miRNA specific primer, RNase inhibitor and reverse transcriptase were added. After being incubated at $25^{\circ} \mathrm{C}$ for $10 \mathrm{~min}, 37^{\circ} \mathrm{C}$ for $50 \mathrm{~min}$ and $70^{\circ} \mathrm{C}$ for $15 \mathrm{~min}$, the cDNA was synthesized and used as a template of qPCR.

miRNA microarray chip analysis. miRNA expression profiling microarray was completed using Agilent human miRNA (8x60K) V18.0 miRNA array (LC Sciences, LLC., Houston, TX, USA). The microarray probe sequence was derived from Sanger MiRBase version 15.0 (http://microrna.sanger. org). Each chip contained multiple quality control probes and employed dual-color chip to examine miRNA expression profiling in MCF-7 cells with overexpressed or normal endogenous expression of SMYD3. Probes were synthesized in situ with photosensitive photogenerated reagents. The sequence consisted of two fragments: A chemically modified oligonucleotide encoding fragment complementary to target miRNA; and an extension arm at the distance specific to the connected encoding sequence that reduced the hybridization spatial impairment. Slides were scanned on an Agilent microarray scanner (model G2565A; Agilent Technologies, Inc., Santa Clara, CA, USA) at 100 and 5\% sensitivity settings. Agilent Feature Extraction software version 8.1 (Agilent Technologies, Inc.) was used for image analysis (10-12).

$R T-q P C R$ of $m R N A$ and miRNA. RT-qPCR was performed using a StepOne ${ }^{\mathrm{TM}}$ Real-Time PCR system (Applied Biosystems; Thermo Fisher Scientific, Inc.). Bestar ${ }^{\circledR}$ SYBR-Green qPCR Mastermix was obtained from DBI Bioscience (http://www.xinghanbio.com/cpzs; Shanghai, China). The thermal profiles were $95^{\circ} \mathrm{C}$ for $10 \mathrm{sec}$ and $60^{\circ} \mathrm{C}$ for $1 \mathrm{~min}$. Melting curve analysis was performed for each PCR to confirm the specificity of amplification. At the end of each phase, fluorescence was measured and quantified. Data is presented as the relative expression level of mRNA following normalization to GAPDH or the relative expression levels of miRNA following normalization to U6 following calculations using the $2^{-\Delta \Delta \mathrm{Cq}}$ method (13). The PCR primer sequences were as follows: GAPDH forward, 5'-ATTCAACGGCACAGTCA AGG-3' and reverse, 5'-GCAGAAGGGGCGGAGATGA-3'; zinc finger E-box binding homeobox (ZEB)1 forward, 5'-AAG GGCAAGAAATCCTGGGG-3' and reverse, 5'-CTCTGGTC CTCTTCAGGTGC-3'; ZEB2 forward, 5'-AAATGCACAGA GTGTGGCAAGG-3' and reverse, 5'-CTGCTGATGTGCGA ACTGTAGGA-3'; SMYD3 forward, 5'-AAGTTCGAACCGC CAAGAG-3' and reverse, 5'-AAGGCAGCGGTCGCAGA CGA-3'; myosin light chain 9 (MYL9) forward, 5'-GAGCCCA AGCGCCTTCT-3' and reverse, 5'-GTCAATGAAGCCATCA CGGT-3'; cysteine rich angiogenic induced 61 (CYR61) forward, 5'-AAGGGGCTGGAATGCAACTT-3' and reverse, 5'-TTGGGGACACAGAGGAATGC-3'; U6 forward, 5'-CTC GCTTCGGCAGCACA-3'; and reverse, 5'-AACGCTTCACG AATTTGCGT-3'; miR200c-3p forward, 5'-ACACTCCAGCT GGGTAATACTGCCGGGTAAT-3'; miR149-3p forward, 5'-ACACTCCAGCTGGGTCTGGCTCCGTGTCTTG-3'; and general reverse, 5'-TGGTGTCGTGGAGTCG-3'. All experiments were repeated three times.

miRNA target gene prediction. In total 5 online prediction software programs, including miRDB (http://mirdb.org/), TargetScan (http://www.targetscan.org/), DIANA-microT (http://diana.cslab.ece.ntua.gr/microT/), microRNA.org (http://www.microrna.org/microrna/home.do) and RNA22 (https://cm.jefferson.edu/rna22), were used to predict the target genes of miRNAs. The intersection of $3 / 5$ of these software programs were selected as the potential target genes.

Statistical analysis. Statistical evaluations were performed using GraphPad Prism (version 5.0; GraphPad Software, Inc., La Jolla, CA, USA), using 3 independent experiments and were analyzed using Student's t-test. $\mathrm{P}<0.05$ was considered to indicate a statistically significant difference. The homologous alignment analysis was performed using DNAMAN (version 6.0; Lynnon LLC., San Ramon, CA, USA) and Primer Premier (version 5.0; Premier Biosoft International, Palo Alto, CA, USA). 


\section{Results}

Effects of SMYD3 overexpression on the miRNA expression profile of $M C F-7$ cells. To investigate the regulatory effects of SMYD3 on global miRNA in breast cancer cells, MCF-7 cells were transfected with SMYD3 and microarray analysis was performed using the Agilent human miRNA (8x60K) V18.0 microarrays chip that contains 1,871 miRNAs. As presented in Fig. 1A, the expression profile analysis identified 1,871 microRNAs with altered expression, including 191 miRNAs that were upregulated in SMYD3-transfected cells compared with mock transfected cells, whilst 1,680 miRNAs were downregulated. Amongst these, 30 miRNAs were upregulated $>2.0$-fold and 24 miRNAs were downregulated $<2.0$-fold (Fig. 1B and C). Furthermore, to investigate the function of these miRNAs in more depth, the potential target genes of these miRNAs and their functions were summarized using bioinformatic analysis (Table I) (14-57).

Verifying the transcriptional regulatory effect of SMYD3 on the target miRNAs, miR-200c-3p and miR149-3p. To confirm the results of miRNA microarray, miR-200c-3p, a predicted target that was upregulated by 37.9-fold, and miR149-3p, a predicted target that was downregulated by 20.9 -fold by SMYD3 in the chip assay, were selected for further investigation. The pcDNA5-TO/TAP-DEST-SMYD3 plasmid and the shSMYD3 plasmid were transfected into MCF-7 cells for the overexpression and knockdown of SMYD3, respectively. In accordance with the results of miRNA microarray, the results of RT-qPCR identified that miR-200c-3p was significantly upregulated following SMYD3 overexpression $(\mathrm{P}<0.001)$ and downregulated following RNA interference (RNAi) -mediated suppression of SMYD3 ( $\mathrm{P}<0.05$; Fig. $2 \mathrm{~A}$ and $\mathrm{B}$ ), whereas the level of miR149-3p exhibited a significant negative association with the expression of SMYD3 (P<0.001; Fig. 2C and D).

The promoter sequence of miR-200c-3p from a number of species, including of Homo sapiens, Macaca mulatta, Pan troglodytes, Monodelphis domestica, Equus caballus and Rattus norvegicus, were analyzed using bioinformatic methods. The results identified that 8 conserved SMYD3 binding sites exist in the promoter region $(-2500$ to +1$)$ of miR-200c-3p from numerous species, indicating that miR-200c-3p may be a target for the transcriptional regulation of SMYD3 (Fig. 3).

Furthermore, the results of RT-qPCR assay demonstrated that the mRNA level of ZEB1 and ZEB2, two predicted target genes of has-miR-200c-3p presented in Table I, was decreased by SMYD3-overexpression, whereas they were increased by RNAi-induced suppression of endogenous SMYD3 (Fig. 4A and B). Taken together, these results demonstrate that SMYD3 activates the transcription of miR-200c-3p and therefore indirectly decreases the mRNA levels of ZEB1/2.

Histone methylation activity is essential in the SMYD3-mediated transactivation of $m i R-200 c-3 p$. Considering the results of the present study and the previous studies that focusing on the histone methylation activity of SMYD3, it was hypothesized that SMYD3 may function as a transactivator of miR-200c-3p via its histone methylation function. To investigate this, MCF-7 cells were treated with $100 \mu \mathrm{M}$ methylthioadenosine, a histone methylation inhibitor, with

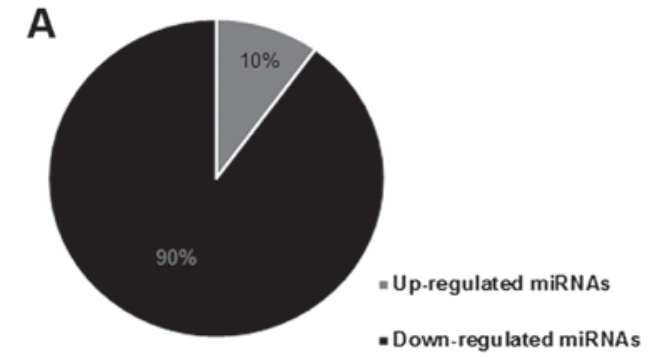

B
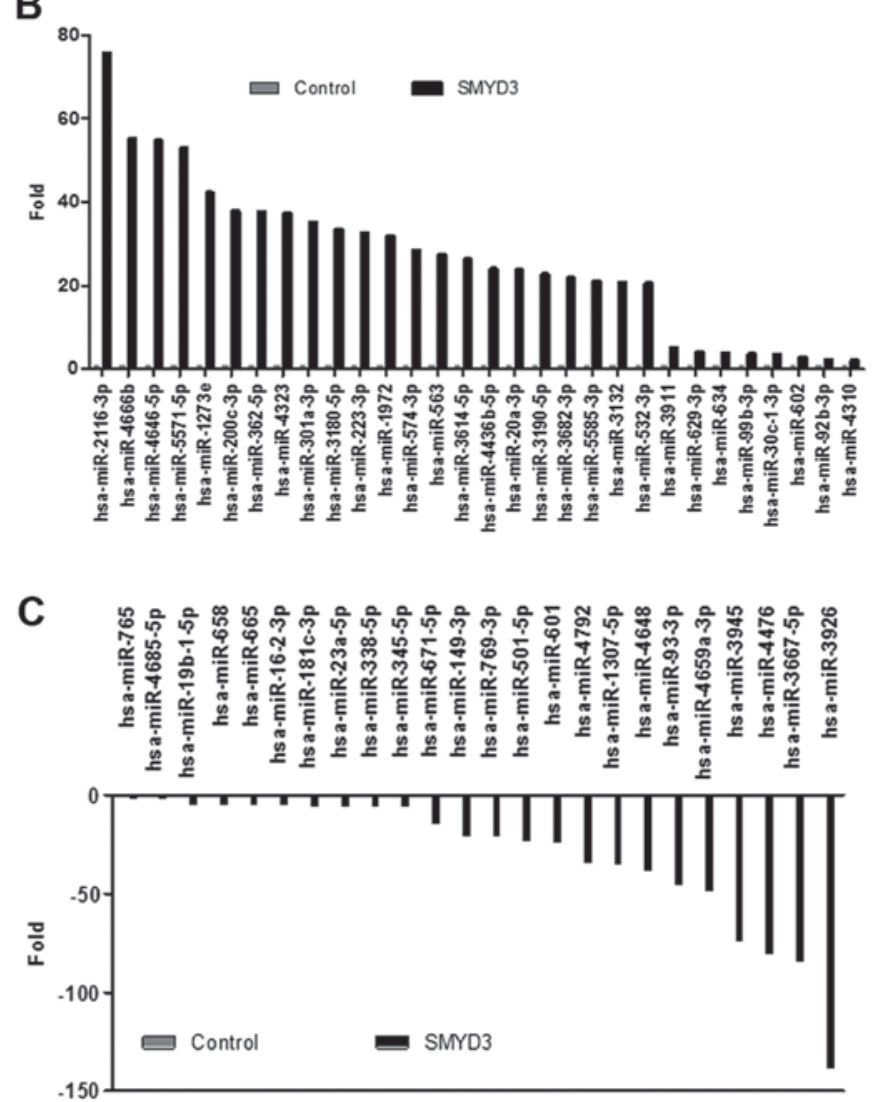

Figure 1. Expression profile of miRNAs following the overexpression of SMYD3 in MCF-7 cells. (A) The percentage of differentially expressed miRNAs following the overexpression of SMYD3 in MCF-7 cells. (B) The 30 miRNAs that were upregulated by $>2.0$-fold. (C) The 24 miRNAs that were downregulated by $>2.0$-fold. miRNA/miR, microRNA; SMYD3, SET and MYND domain containing 3.

or without physiological concentration of estradiol $\left(10^{-11} \mathrm{M}\right)$ and subsequently the transcription level of miR-200c-3p was detected using RT-qPCR. As presented in Fig. 5, the transcription level of miR-200c-3p was significantly downregulated by the histone methylation inhibitor (Fig. 5A). Consistently, SMYD3- $\mathrm{N}$ HSC, a histone methyltransferase-activity-depleted mutant of SMYD3 $(58,59)$, also decreases the transcription level of miR-200c-3p (Fig. 5B). These results suggested that the histone methylation activity may have vital roles in SMYD3-mediated transactivation of miR-200c-3p.

Regulatory effects of SMYD3 and miR-200c-3p on target genes of the RhoA-myocardin-related transcription factor A (MRTF-A) signaling pathway. The authors' previous study demonstrated that SMYD3 promotes MRTF-A-mediated transactivation of MYL9 and migration of MCF-7 breast 


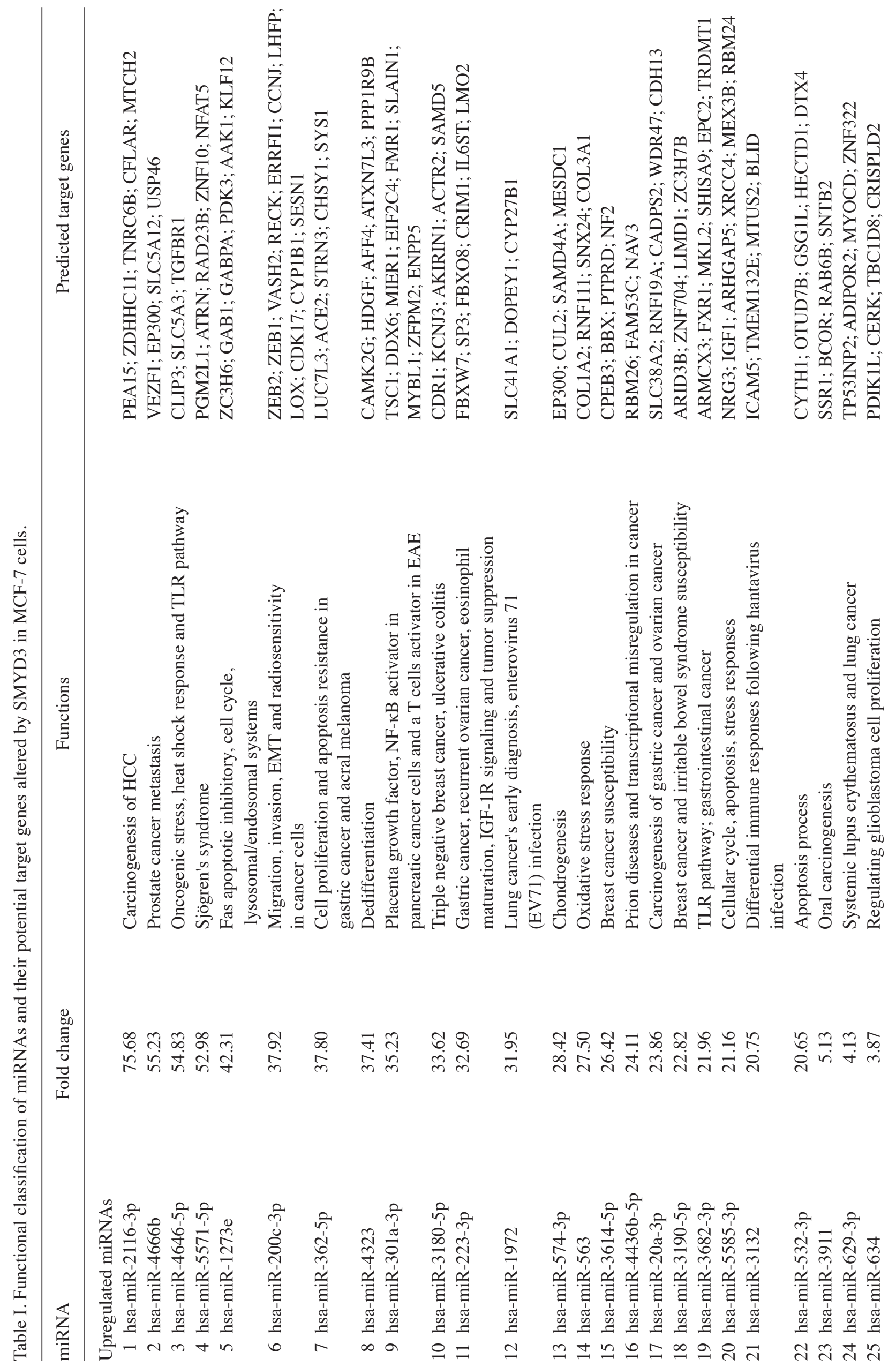



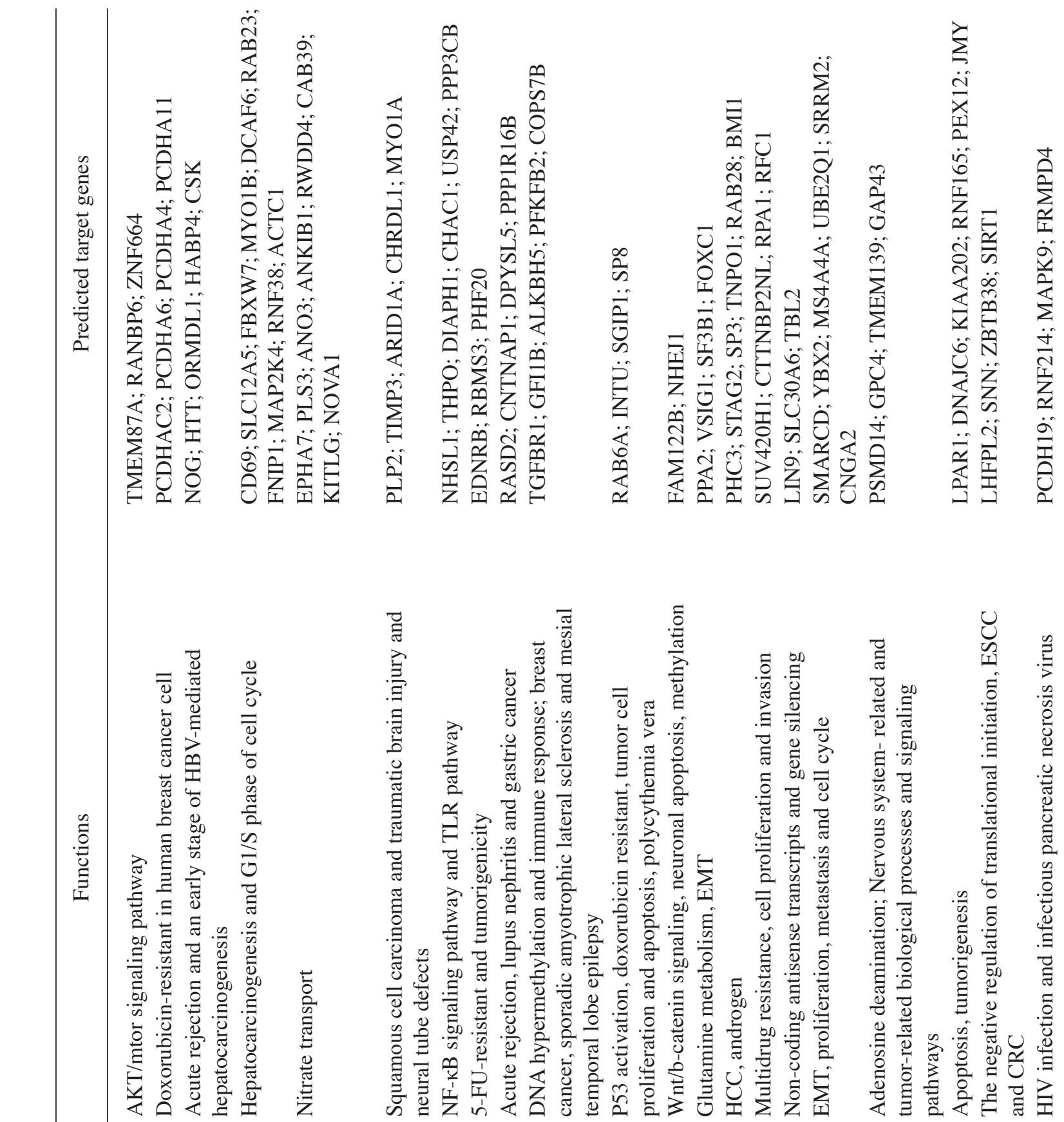

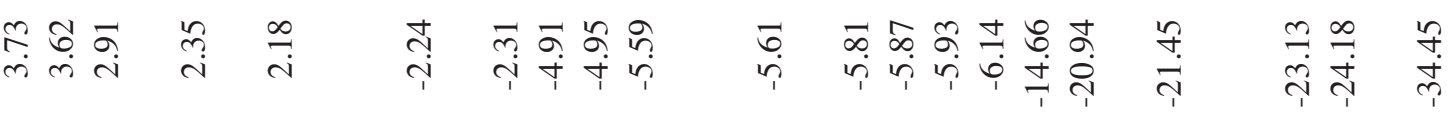

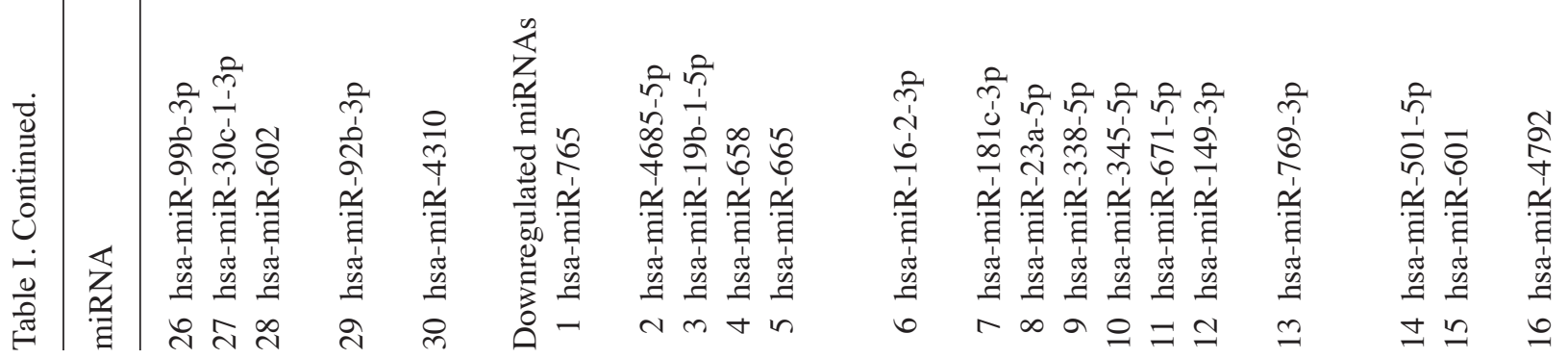




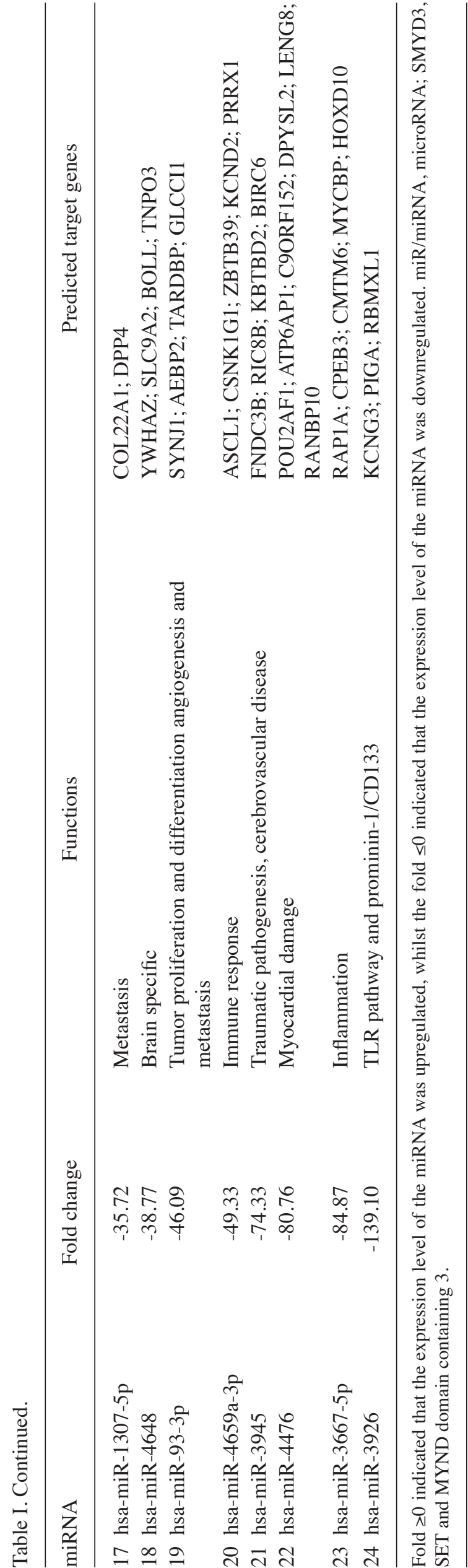

cancer cells (59). However, other previous studies have identified that miR-200c represses migration and invasion of breast cancer cells via suppression of the RhoA-MRTF-A signaling pathway $(60,61)$. As the results of the present study demonstrated the overexpression of SMYD3 directly transactivates miR-200c-3p, whether SMYD3 and miR-200c-3p supports or opposes each others' effects on the transcription of MYL9 and CYR61, two MRTF-A-dependent migration-related genes, was investigated. The miR-200c-3p mimic and/or SMYD3-overexpression plasmid were transfected into MCF7 cells and the transcription level of MYL9 and CYR61 were detected using RT-qPCR. The results of RT-qPCR demonstrated that the transcriptional level of SMYD3 and the miR-200c-3p increased significantly following the transfection (Fig. 6A and B). As presented in Fig. 6C, the mRNA levels of MYL9 and CYR61 were suppressed by miR-200c-3p and increased by the overexpression of SMYD3. Furthermore, the upregulation of MYL9 and CYR61 remained after SMYD3 was co-transfected with miR-200c-3p mimics, but the effect in combination group was reduced compared with that of the SMYD3-transfected group. These results demonstrated that miR-200c-3p may be a downstream negative regulator of the SMYD3-mediated pathway in the migration of breast cancer cells.

\section{Discussion}

The primary focus of epigenetics is to elucidate the heritable changes in gene expression and regulation, which occur without mutation to the DNA sequence, in the process of gene transcription in eukaryotic cells. The heritable alterations include DNA methylation, histone modification and regulation by noncoding RNAs. A number of studies have established that the majority of malignant tumor types exhibit cancer-associated epigenetics alterations (62). However, the interdisciplinary investigation of miRNA and histone modification has received increasing interest.

A previous study has analyzed downstream coding genes of SMYD3 using cDNA microarray and the results identified that there were 61 genes upregulated $>3$-fold and genes downregulated $<3$-fold by SMYD3 (1). However, to date, the downstream miRNAs regulated by SMYD3 are yet to be elucidated. Therefore, the present study focused on the effect of SMYD3 on the miRNA expression profile in MCF-7 breast cancer cells by analyzing microarray and RT-qPCR data. These results demonstrated that 30 miRNAs were upregulated $>2.0$-fold, whilst 24 miRNAs were downregulated $<2.0$-fold following the overexpression of SMYD3. The analysis of bioinformatic and previously published data identified that the majority of these potential downstream miRNAs of SMYD3 were associated with the proliferation, migration and therapy resistance of tumor.

To further validate the results of microarray, RT-qPCR was performed to detect the effects of SMYD3 on the expression of miR-200c-3p and miR-149, which are 2 representative target miRNAs of SMYD3 with opposing alterations, and the results were in accordance with those of the microarray. Previous studies have demonstrated that miR-149 and miR-200c are associated with the progression of cancer (63-65). miR-149 has been established as a tumor suppressor by inhibiting the spreading, migration and invasion of basal-like breast cancer 

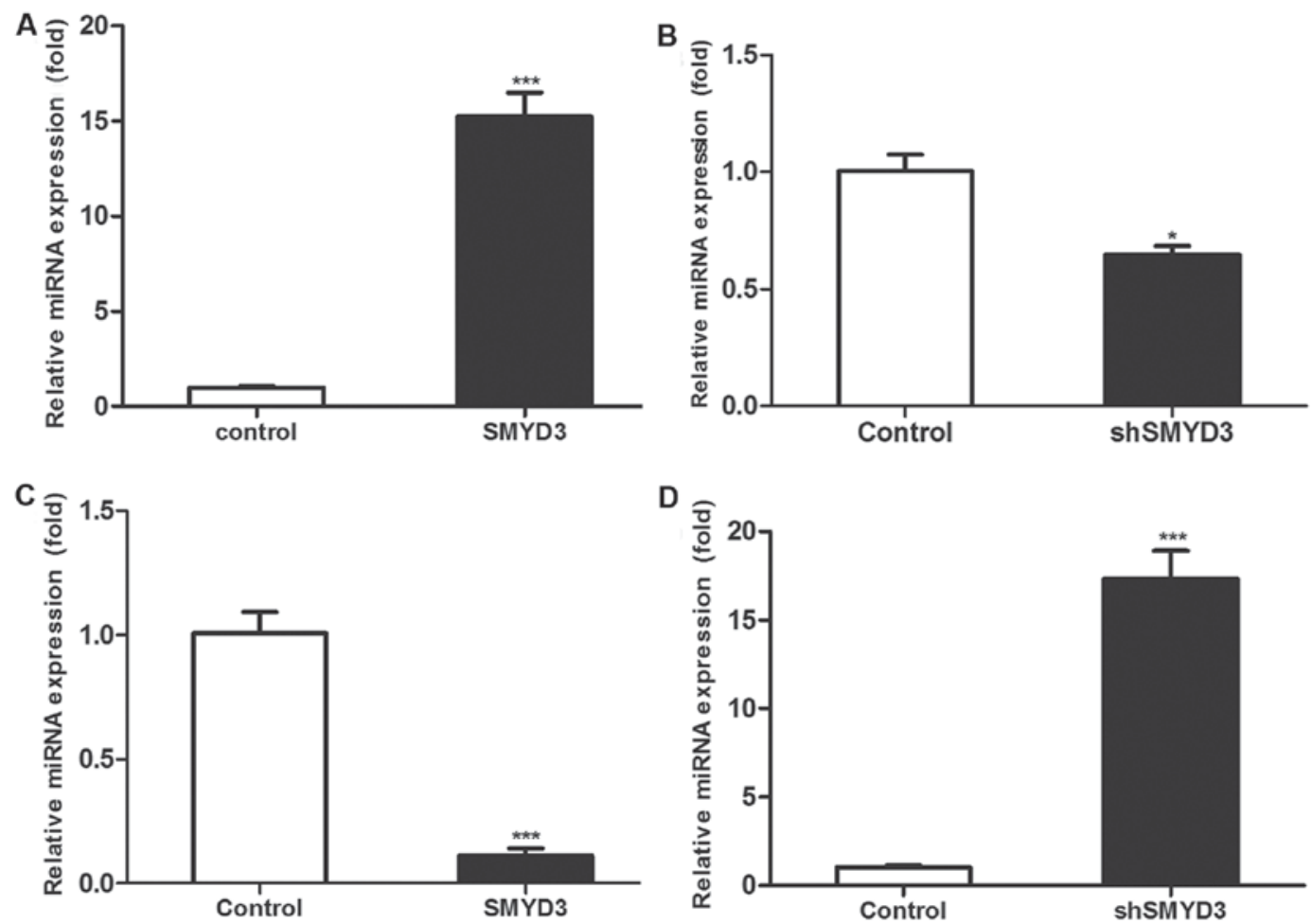

Figure 2. SMYD3 upregulates miR-200c-3p and downregulates miR149-3p in MCF-7 cells. The transcriptional levels of (A and B) miR-200c-3p and (C and D) miR149-3p in MCF-7 cells transfected with pcDNA5-TO/TAP-DEST-SMYD3 and shSMYD3 was detected using reverse transcription-quantitative polymerase chain reaction. ${ }^{*} \mathrm{P}<0.05,{ }^{* * * *} \mathrm{P}<0.001$. miRNA/miR, microRNA; SMYD3, SET and MYND domain containing 3; sh, short hairpin.

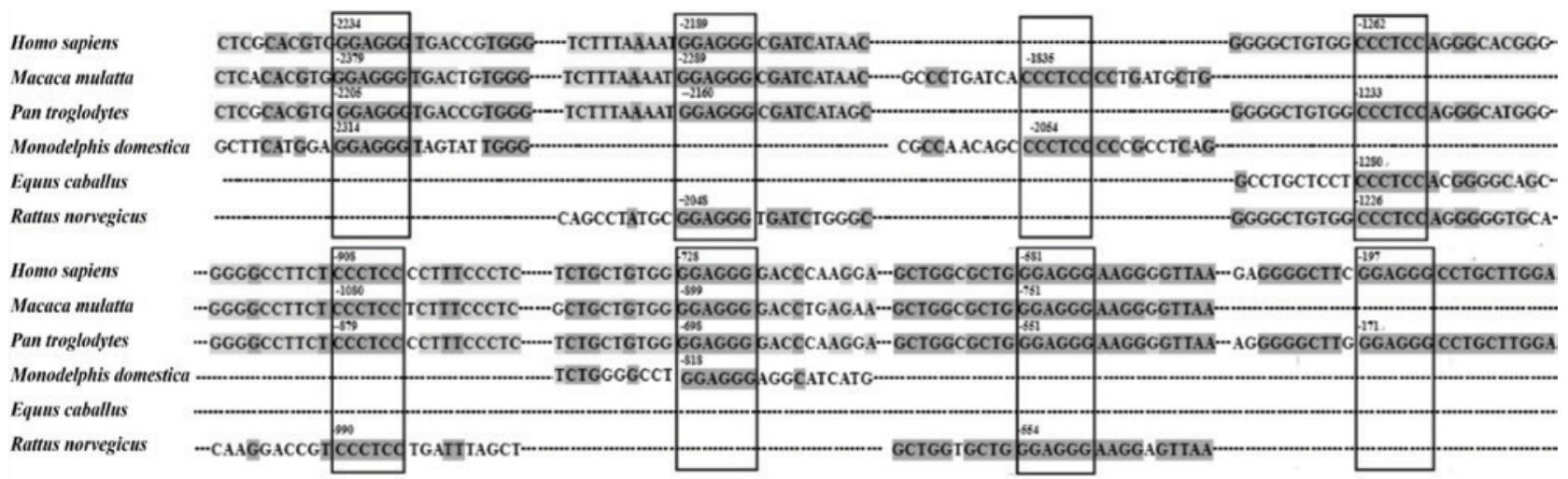

Figure 3. Conserved SMYD3 binding sites in miR-200c-3p promoter from numerous species. The homologous alignment analysis of the binding sides of SMYD3 in miR-200c-3p promoter from a number of species was performed using DNAMAN (version 6.0) and Primer Premier (version 5.0). miR, microRNA; SMYD3, SET and MYND domain containing 3.

cells (63). However, the roles of miR-200c in cancer remain to be fully understood. A number of previous studies have reported that miR-200c may inhibit the epithelial-mesenchymal transition and enhance the chemosensitivity and radiosensitivity of cancer cells $(64,65)$, whereas another previous study reported a metastasis-promoting role of miR-200 in breast cancer (64). In addition, a separate study demonstrated that the expression level of miR-200c different between the subtypes of breast cancer (65). Compared with the normal breast epithelial cells (MCF-10A), the basal cancer cells (MDA-MB-231 and BT549) exhibited a reduced expression of miR-200c (65). However, the expression of miR-200c in luminal cancer cells (MCF-7 and BT474) was increased compared with that in the normal breast epithelial cells (65). Additionally, previous studies have suggested that miR-200c may repress migration and invasion of breast cancer cells via the suppression of RhoA-MRTF-A signaling pathways $(60,61)$, whereas the current study suggests that SMYD3 promotes MRTF-A-mediated transactivation of MYL9 and migration of MCF-7 breast cancer cells (59). The results in the present study have demonstrated that the expression of miR-200c in MCF-7 breast cancer cells may be upregulated by SMYD3, and the overexpression of miR-200c partially inhibits the transactivation effects of SMYD3 on the MRTF-A-dependent migration-associated genes. Taken together, these results indicated that miR-200c may be a downstream negative regulator of the SMYD3-mediated pathway in the migration of breast cancer cells, and may promote a negative feedback loop to prevent excessive induction of migration 

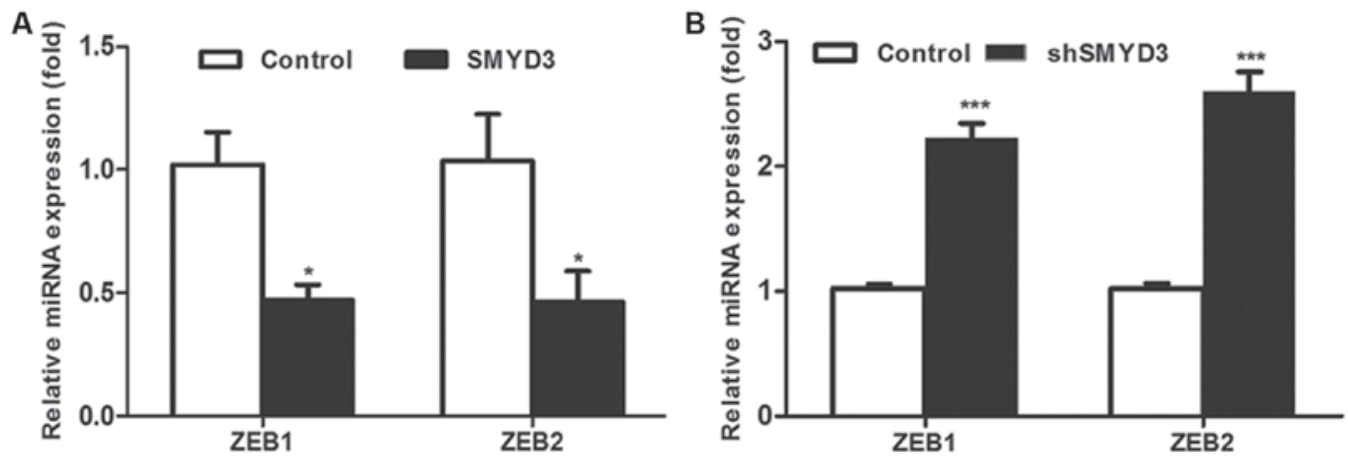

Figure 4. SMYD3 decreases the mRNA level of ZEB1/2, 2 predicted target genes of miR-200c-3p. (A) The mRNA level of ZEB1/2 in MCF-7 cells transfected with pcDNA5-TO/TAP-DEST-SMYD3 plasmid was detected using RT-qPCR. (B) The mRNA level of ZEB1/2 in MCF-7 cells transfected with shSMYD3 plasmid was detected using RT-qPCR. ${ }^{*} \mathrm{P}<0.05,{ }^{* * * *} \mathrm{P}<0.001$. miR/miRNA, microRNA; SMYD3, SET and MYND domain containing 3; ZEB, zinc finger E-box binding homeobox; sh, short hairpin; RT-qPCR, reverse transcription-quantitative polymerase chain reaction.
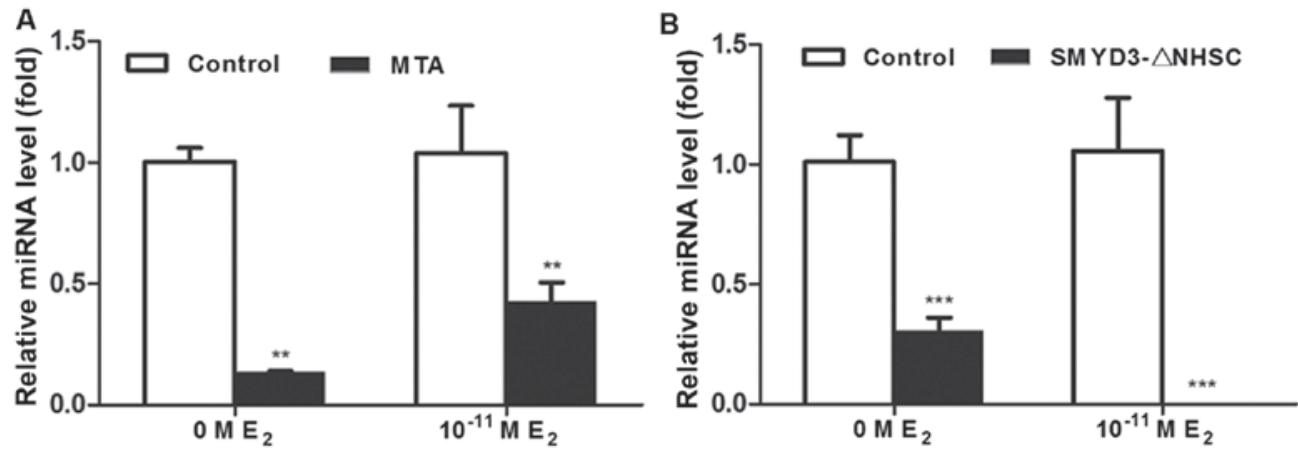

Figure 5. Histone methylation activity of SMYD3 has vital roles in promoting miR-200c-3p. (A) The difference of miR-200c-3p levels in MCF-7 cells treated by MTA with or without $\mathrm{E}_{2}\left(10^{-11} \mathrm{M}\right)$ was detected by RT-qPCR. (B) The difference of miR-200c-3p levels in MCF-7 cells transfected with SMYD3- $\triangle$ NHSC (Asn-His-Ser-Cys) plasmid and treated with or without $\mathrm{E}_{2}\left(10^{-11} \mathrm{M}\right)$ was detected by RT-qPCR. ${ }^{* *} \mathrm{P}<0.01,{ }^{* * * *} \mathrm{P}<0.001$. miR/miRNA, microRNA; SMYD3, SET and MYND domain containing 3; RT-qPCR, reverse transcription-quantitative polymerase chain reaction; MTA, methylthioadenosine; $\mathrm{E}_{2}$, estradiol.
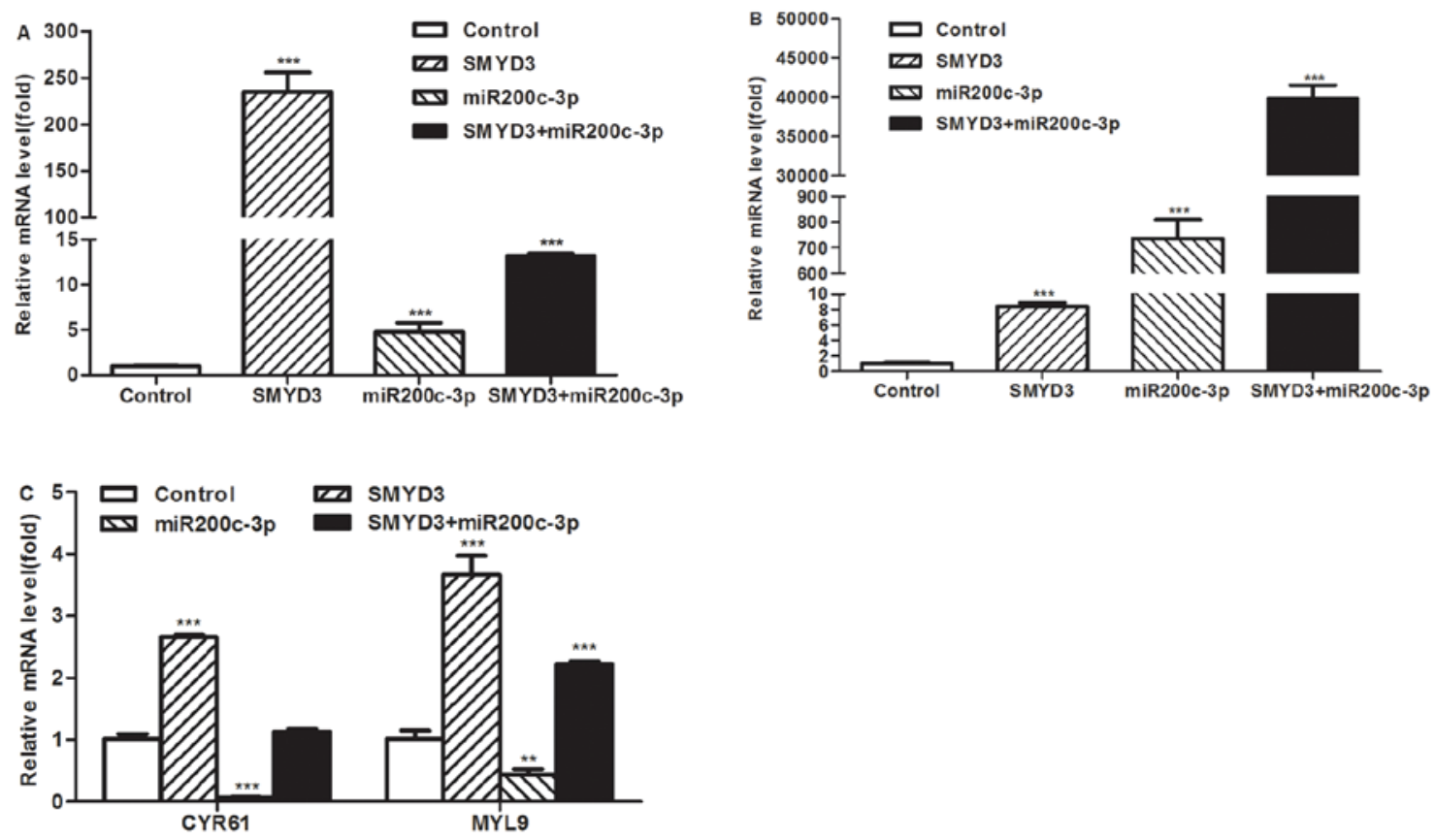

Figure 6. miR-200c-3p may be a downstream negative regulator of SMYD3-mediated pathway. (A) The transfection efficiency of SMYD3 was detected using RT-qPCR. (B) The transfection efficiency of miR-200c-3p mimics was detected using RT-qPCR. (C) miR-200c-3p mimics and/or SMYD3-overespression plasmids were transfected into MCF7 cells and the transcription levels of MYL9 and CYR61 were detected using RT-qPCR. ${ }^{* *} \mathrm{P}<0.01,{ }^{* * *} \mathrm{P}<0.001$. miR/miRNA, microRNA; SMYD3, SET and MYND domain containing 3; RT-qPCR, reverse transcription-quantitative polymerase chain reaction; CYR61, cysteine rich angiogenic induced 61; MYL9, myosin light chain. 
of breast cancer cells. However, the underlying mechanism of miR-200c in breast cancer remains to be elucidated by future investigation.

Additionally, previous studies have identified that SMYD3 alters chromatin structure by catalyzing the di-/tri-methylation of histone $\mathrm{H} 3$ at lysine 4 (H3K4me2/3), H4K20me 3 and H4K5me $(5,6)$. As part of the established methylation marks at H3K4 or H3K36, mono-methylations of H3K9, H3K27, H3K79, $\mathrm{H} 4 \mathrm{~K} 20$ and $\mathrm{H} 2 \mathrm{BK} 5$ are associated with transcriptional activation, whereas trimethylations of H3K9, H3K27, H3K79 and H4K 20 are associated with transcriptional repression $(5,6)$. The current study demonstrates that the histone methylation activity is essential for SMYD3-mediated transactivation of miR-200c-3p. Therefore, SMYD3 antagonistic regulation of the downstream miRNAs may also depend on the distinct modification on the histone adjacent to the promoter.

In conclusion, the effect of SMYD3 on the miRNA expression profile in MCF-7 breast cancer cells was analyzed using microarray and RT-qPCR in the present study. To the best of our knowledge, this is the first study focused on the transcriptional regulation of SMYD3 on miRNAs. These results may provide a novel theoretical basis to elucidate the mechanism underlying the initiation, progression, diagnose, prevention and therapy of breast cancer.

\section{Acknowledgements}

The current study was supported by grants from the National Natural Science Foundation of China (grant no. 31470816 , 31300642 and 31000343), the College Students' Innovation and Entrepreneurship Training Program of Tianjin (grant no. 201510057057) and the Program for Changjiang Scholars and Innovative Research Team in University of Ministry of Education of China (grant no. IRT1166).

\section{References}

1. Hamamoto R, Furukawa Y, Morita M, Iimura Y, Silva FP, Li M, Yagyu R and Nakamura Y: SMYD3 encodes a histone methyltransferase involved in the proliferation of cancer cells. Nat Cell Biol 6: 731-740, 2004.

2. Hamamoto R, Silva FP, Tsuge M, Nishidate T, Katagiri T, Nakamura Y and Furukawa Y: Enhanced SMYD3 expression is essential for the growth of breast cancer cells. Cancer Sci 97: $113-118,2006$.

3. Wang SZ, Luo XG, Shen J, Zou JN, Lu YH and Xi T: Knockdown of SMYD3 by RNA interference inhibits cervical carcinoma cell growth and invasion in vitro. BMB Rep 41: 294-299, 2008.

4. Liu Y, Luo X, Deng J, Pan Y, Zhang L and Liang H: SMYD3 overexpression was a risk factor in the biological behavior and prognosis of gastric carcinoma. Tumour Biol 36: 2685-2694, 2015.

5. Van Aller GS, Reynoird N, Barbash O, Huddleston M, Liu S, Zmoos AF, McDevitt P, Sinnamon R, Le B, Mas G, et al: Smyd3 regulates cancer cell phenotypes and catalyzes histone $\mathrm{H} 4$ lysine 5 methylation. Epigenetics 7: 340-343, 2012.

6. Foreman KW, Brown M, Park F, Emtage S, Harriss J, Das C, Zhu L, Crew A, Arnold L, Shaaban S and Tucker P: Structural and functional profiling of the human histone methyltransferase SMYD3. PloS One 6: e22290, 2011.

7. Ambros V: The functions of animal microRNAs. Nature 431: 350-355, 2004.

8. Yahya SM and Elsayed GH: A summary for molecular regulations of miRNAs in breast cancer. Clin Biochem 48: 388-396, 2015.

9. Zhang L, Deng L, Chen F, Yao Y, Wu B, Wei L, Mo Q and Song Y: Inhibition of histone H3K 79 methylation selectively inhibits proliferation, self-renewal and metastatic potential of breast cancer. Oncotarget 5: 10665-10677, 2014.
10. Wang H, Ach RA and Curry B: Direct and sensitive miRNA profiling from low-input total RNA. RNA 13: 151-159, 2007.

11. Hughes TR, Mao M, Jones AR, Burchard J, Marton MJ, Shannon KW, Lefkowitz SM, Ziman M, Schelter JM, Meyer MR, et al: Expression profiling using microarrays fabricated by an ink-jet oligonucleotide synthesizer. Nature Biotechnol 19: 342-347, 2001.

12. Dennis G Jr, Sherman BT, Hosack DA, Yang J, Gao W, Lane HC and Lempicki RA: DAVID: Database for annotation, visualization and integrated discovery. Genome Biol 4: P3, 2003.

13. Livak KJ and Schmittgen TD: Analysis of relative gene expression data using real-time quantitative PCR and the 2(-Delta Delta C(T)) method. Methods 25: 402-408, 2001.

14. Katayama Y, Maeda M, Miyaguchi K, Nemoto S, Yasen M, Tanaka S, Mizushima H, Fukuoka Y, Arii S and Tanaka H: Identification of pathogenesis-related microRNAs in hepatocellular carcinoma by expression profiling. Oncology Lett 4: 817-823, 2012.

15. Watahiki A, Wang Y, Morris J, Dennis K, O'Dwyer HM, Gleave M, Gout PW and Wang Y: MicroRNAs associated with metastatic prostate cancer. PloS One 6: e24950, 2011.

16. Moossavi S and Rezaei N: Toll-like receptor signalling and their therapeutic targeting in colorectal cancer. Int Immunopharmacol 16: 199-209, 2013.

17. Place RF and Noonan EJ: Non-coding RNAs turn up the heat: An emerging layer of novel regulators in the mammalian heat shock response. Cell Stress Chaperones 19: 159-172, 2014.

18. Li J, Tan Q, Yan M, Liu L, Lin H, Zhao F, Bao G, Kong H, Ge C, Zhang F, et al: miRNA-200c inhibits invasion and metastasis of human non-small cell lung cancer by directly targeting ubiquitin specific peptidase 25. Mol Cancer 13: 166, 2014.

19. Shi L, Fei X, Sun G, Wang Z, Wan Y, Zeng Y and Guo J: Hypothermia stimulates glioma stem spheres to spontaneously dedifferentiate adjacent non-stem glioma cells. Cell Mol Neurobiol 35: 217-230, 2015.

20. Mycko MP, Cichalewska M, Machlanska A, Cwiklinska H, Mariasiewicz M and Selmaj KW: MicroRNA-301a regulation of a T-helper 17 immune response controls autoimmune demyelination. Proc Natl Acad Sci USA 109: E1248-E1257, 2012.

21. Archanioti P, Gazouli M, Theodoropoulos G, Vaiopoulou A and Nikiteas N: Micro-RNAs as regulators and possible diagnostic bio-markers in inflammatory bowel disease. J Crohns Colitis 5: 520-524, 2011.

22. Li BS, Zhao YL, Guo G, Li W, Zhu ED, Luo X, Mao XH, Zou QM, Yu PW, Zuo QF, et al: Plasma microRNAs, miR-223, miR-21 and miR-218, as novel potential biomarkers for gastric cancer detection. PLoS One 7: e41629, 2012.

23. Madhavan D, Cuk K, Burwinkel B and Yang R: Cancer diagnosis and prognosis decoded by blood-based circulating microRNA signatures. Front Genet 4: 116, 2013.

24. Guerit D, Philipot D, Chuchana P, Toupet K, Brondello JM, Mathieu M, Jorgensen C and Noël D: Sox9-regulated miRNA-574-3p inhibits chondrogenic differentiation of mesenchymal stem cells. PLoS One 8: e62582, 2013.

25. Cheung O, Puri P, Eicken C, Contos MJ, Mirshahi F, Maher JW, Kellum JM, Min H, Luketic VA and Sanyal AJ: Nonalcoholic steatohepatitis is associated with altered hepatic MicroRNA expression. Hepatology 48: 1810-1820, 2008.

26. Marrale M, Albanese NN, Cali F and Romano V: Assessing the impact of copy number variants on miRNA genes in autism by Monte Carlo simulation. PLoS One 9: e90947, 2014.

27. Li X, Zhang Z, Yu M, Li L, Du G, Xiao W and Yang H: Involvement of miR-20a in promoting gastric cancer progression by targeting early growth response 2 (EGR2). Int J Mol Sci 14: $16226-16239,2013$.

28. Fan X, Liu Y, Jiang J, Ma Z, Wu H, Liu T, Liu M, Li X and Tang H: miR-20a promotes proliferation and invasion by targeting APP in human ovarian cancer cells. Acta Biochim Biophys Sin (Shanghai) 42: 318-324, 2010.

29. Ivashchenko A, Berillo O, Pyrkova A, Niyazova R and Atambayeva S: The properties of binding sites of miR-619-5p, miR-5095, miR-5096, and miR-5585-3p in the mRNAs of human genes. Biomed Res Int 2014: 720715, 2014.

30. Shin OS, Kumar M, Yanagihara R and Song JW: Hantaviruses induce cell type- and viral species-specific host microRNA expression signatures. Virology 446: 217-224, 2013.

31. Cheng Y, Kuang W, Hao Y,Zhang D, Lei M, Du L, Jiao H, Zhang X and Wang F: Downregulation of miR-27a* and miR-532-5p and upregulation of miR-146a and miR-155 in LPS-induced RAW264.7 macrophage cells. Inflammation 35: 1308-1313, 2012. 
32. Lu MC, Lai NS, Chen HC, Yu HC, Huang KY, Tung CH, Huang HB and Yu CL: Decreased microRNA(miR)-145 and increased miR-224 expression in $\mathrm{T}$ cells from patients with systemic lupus erythematosus involved in lupus immunopathogenesis. Clin Exp Immunol 171: 91-99, 2013.

33. Jeansonne D, Pacifici M, Lassak A, Reiss K, Russo G, Zabaleta J and Peruzzi F: Differential effects of microRNAs on glioblastoma growth and migration. Genes (Basel) 4: 46-64, 2013.

34. Jin Y, Tymen SD, Chen D, Fang ZJ, Zhao Y, Dragas D, Dai Y, Marucha PT and Zhou X: MicroRNA-99 family targets AKT/mTOR signaling pathway in dermal wound healing. PLoS One 8: e64434, 2013.

35. Fang Y, Shen H, Cao Y, Li H, Qin R, Chen Q, Long L, Zhu XL, $\mathrm{Xie} \mathrm{CJ}$ and $\mathrm{Xu} \mathrm{WL}$ : Involvement of miR-30c in resistance to doxorubicin by regulating YWHAZ in breast cancer cells. Braz J Med Biol Res 47: 60-69, 2014.

36. Sengupta S, Nie J, Wagner RJ, Yang C, Stewart R and Thomson JA: MicroRNA 92b controls the G1/S checkpoint gene p57 in human embryonic stem cells. Stem Cells 27: 1524-1528, 2009.

37. Balakathiresan N, Bhomia M, Chandran R, Chavko M, McCarron RM and Maheshwari RK: MicroRNA let-7i is a promising serum biomarker for blast-induced traumatic brain injury. J Neurotrauma 29: 1379-1387, 2012.

38. Kurokawa K, Tanahashi T, Iima T, Yamamoto Y, Akaike Y, Nishida K, Masuda K, Kuwano Y, Murakami Y, Fukushima M and Rokutan K: Role of miR-19b and its target mRNAs in 5-fluorouracil resistance in colon cancer cells. J Gastroenterol 47: 883-895, 2012

39. Hackl M, Brunner S, Fortschegger K, Schreiner C, Micutkova L, Mück C, Laschober GT, Lepperdinger G, Sampson N, Berger P, et al: miR-17, miR-19b, miR-20a, and miR-106a are down-regulated in human aging. Aging Cell 9: 291-296, 2010.

40. Sui W, Dai Y, Huang Y, Lan H, Yan Q and Huang H: Microarray analysis of MicroRNA expression in acute rejection after renal transplantation. Transpl Immunol 19: 81-85, 2008.

41. Tsuruta T, Kozaki K, Uesugi A, Furuta M, Hirasawa A, Imoto I, Susumu N, Aoki D and Inazawa J: miR-152 is a tumor suppressor microRNA that is silenced by DNA hypermethylation in endometrial cancer. Cancer Res 71: 6450-6462, 2011

42. Lezina L, Purmessur N, Antonov AV, Ivanova T, Karpova E, Krishan K, Ivan M, Aksenova V, Tentler D, Garabadgiu AV, et al: miR-16 and miR-26a target checkpoint kinases Weel and Chk1 in response to p53 activation by genotoxic stress. Cell Death Dis 4: e953, 2013.

43. Ciafre SA, Galardi S, Mangiola A, Ferracin M, Liu CG Sabatino G, Negrini M, Maira G, Croce CM and Farace MG: Extensive modulation of a set of microRNAs in primary glioblastoma. Biochem Biophys Res Commun 334: 1351-1358, 2005.

44. Rathore MG, Saumet A, Rossi JF, de Bettignies C, Tempé D, Lecellier $\mathrm{CH}$ and Villalba M: The NF- $\kappa \mathrm{B}$ member p65 controls glutamine metabolism through miR-23a. Int J Biochem Cell Biol 44: 1448-1456, 2012.

45. Huang XH, Wang Q, Chen JS, Fu XH, Chen XL, Chen LZ, Li W, Bi J, Zhang LJ, Fu Q, et al: Bead-based microarray analysis of microRNA expression in hepatocellular carcinoma: miR-338 is downregulated. Hepatol Res 39: 786-794, 2009.

46. Schou JV, Rossi S, Jensen BV, Nielsen DL, Pfeiffer P, Høgdall E, Yilmaz M, Tejpar S, Delorenzi M, Kruhøffer M and Johansen JS miR-345 in metastatic colorectal cancer: A non-invasive biomarker for clinical outcome in non-KRAS mutant patients treated with 3rd line cetuximab and irinotecan. PloS One 9: e99886, 2014.

47. Rossi JJ: A novel nuclear miRNA mediated modulation of a non-coding antisense RNA and its cognate sense coding mRNA. EMBO J 30: 4340-4341, 2011

48. Ke Y,Zhao W,Xiong J and Cao R: miR-149 inhibits non-small-cell lung cancer cells EMT by targeting FOXM1. Biochem Res Int 2013: 506731, 2013.
49. Liu F, Xiong Y, Zhao Y, Tao L, Zhang Z, Zhang H, Liu Y, Feng G, $\mathrm{Li} \mathrm{B}, \mathrm{He} \mathrm{L}$, et al: Identification of aberrant microRNA expression pattern in pediatric gliomas by microarray. Diagn Pathol 8: 158, 2013.

50. Sun Y, Wu J, Wu SH, Thakur A, Bollig A, Huang Y and Liao DJ: Expression profile of microRNAs in c-Myc induced mouse mammary tumors. Breast Cancer Res Treat 118: 185-196, 2009.

51. Ohdaira H, Nakagawa $\mathrm{H}$ and Yoshida K: Profiling of molecular pathways regulated by microRNA 601. Comput Biol Chem 33: 429-433, 2009.

52. Chang ST, Thomas MJ, Sova P, Green RR, Palermo RE and Katze MG: Next-generation sequencing of small RNAs from HIV-infected cells identifies phased microrna expression patterns and candidate novel microRNAs differentially expressed upon infection. MBio 4: e00549-e00512, 2013.

53. Du L, Schageman JJ, Subauste MC, Saber B, Hammond SM, Prudkin L, Wistuba II, Ji L, Roth JA, Minna JD and Pertsemlidis A: miR-93, miR-98, and miR-197 regulate expression of tumor suppressor gene FUS1. Mol Cancer Res 7: 1234-1243, 2009.

54. Asghari Alashti F and Minuchehr Z: MiRNAs which target CD3 subunits could be potential biomarkers for cancers. PLoS One 8: e78790, 2013

55. Yang J, Liang Y, Han $\mathrm{H}$ and Qin H: Identification of a miRNA signature in neutrophils after traumatic injury. Acta Biochim Biophys Sin (Shanghai) 45: 938-945, 2013.

56. Rustagi Y and Rani V: Screening of microRNA as potential CardiomiRs in Rattus noveregicus heart related dataset. Bioinformation 9: 919-922, 2013.

57. Rappa G, Mercapide J, Anzanello F, Pope RM and Lorico A: Biochemical and biological characterization of exosomes containing prominin-1/CD133. Mol Cancer 12: 62, 2013.

58. Kim H, Heo K, Kim JH, Kim K, Choi J and An W: Requirement of histone methyltransferase SMYD3 for estrogen receptor-mediated transcription. J Biol Chem 284: 19867-19877, 2009.

59. Luo XG, Zhang CL, Zhao WW, Liu ZP, Liu L, Mu A, Guo S, Wang N, Zhou H and Zhang TC: Histone methyltransferase SMYD3 promotes MRTF-A-mediated transactivation of MYL9 and migration of MCF-7 breast cancer cells. Cancer Lett 344: 129-137, 2014.

60. Jurmeister S, Baumann M, Balwierz A, Keklikoglou I, Ward A, Uhlmann S, Zhang JD, Wiemann S and Sahin Ö: MicroRNA-200c represses migration and invasion of breast cancer cells by targeting actin-regulatory proteins FHOD1 and PPM1F. Mol Cell Biol 32: 633-651, 2012.

61. Wu XD, Liu WL, Zeng K, Lei HY, Zhang QG, Zhou SQ and Xu SY: Advanced glycation end products activate the miRNA/RhoA/ROCK2 pathway in endothelial cells. Microcirculation 21: 178-186, 2014.

62. Cava C, Bertoli G and Castiglioni I: Integrating genetics and epigenetics in breast cancer: Biological insights, experimental, computational methods and therapeutic potential. BMC Syst Biol 9: 62, 2015.

63. Bischoff A, Huck B, Keller B, Strotbek M, Schmid S, Boerries M, Busch H, Müller D and Olayioye MA: miR149 functions as a tumor suppressor by controlling breast epithelial cell migration and invasion. Cancer Res 74: 5256-5265, 2014.

64. Zhang HF, Xu LY and Li EM: A family of pleiotropically acting microRNAs in cancer progression, miR-200: Potential cancer therapeutic targets. Curr Pharm Des 20: 1896-1903, 2014

65. Sun Q, Liu T, Yuan Y, Guo Z, Xie G, Du S, Lin X, Xu Z, Liu M, Wang W, et al: MiR-200c inhibits autophagy and enhances radiosensitivity in breast cancer cells by targeting UBQLN1. Int J Cancer 136: 1003-1012, 2015. 